\title{
Is the common carotid artery intima-media thickness associated with functional outcome after acute ischaemic stroke?
}

\author{
J Ellul, P Talelli, G Terzis, A Chrysanthopoulou, G Gioldasis, T Papapetropoulos
}

J Neurol Neurosurg Psychiatry 2004;75:1197-1 199. doi: 10.1136/jnnp.2003.017558

Background: Common carotid artery intima-media thickness (CCA-IMT) is an independent and early marker of generalised atherosclerosis. Brain affected by atherosclerosis may be more vulnerable to an ischaemic insult.

Objective: To investigate the association between CCA-IMT and functional outcome after an acute ischaemic stroke.

Design: Prospective cohort analysis.

Methods: 284 consecutive patients (mean (SD) age, 68.7 (12.7) years, $126(44 \%)$ female) with an acute ischaemic stroke had carotid ultrasonography, carried out by a single operator. Demographic data, vascular risk factors, initial stroke severity, and brain imaging findings were recorded. Outcome was assessed at seven days from stroke onset, at discharge from hospital, and at one year post-stroke.

Results: CCA-IMT was not significantly associated with adverse short or long term functional outcome in univariate analysis, or after adjustment in a multivariate logistic regression analysis for demographic data, initial stroke severity, conventional vascular risk factors, and the characteristics of the ischaemic lesion. Age and initial stroke severity were the only independent predictors of outcome. Conclusions: CCA-IMT was not associated with adverse functional outcome after an ischaemic stroke. Adding CCAIMT in a prediction model for stroke outcome would probably not improve the power of the model.

\footnotetext{
C
} ommon carotid artery intima-media thickness (CCAIMT) has been identified as an independent and early marker of generalised atherosclerosis, and is associated with increased risk of stroke. ${ }^{1-3}$ Raised values of CCA-IMT are associated with the presence of certain cardiovascular risk factors in both stroke patients and controls. ${ }^{12}$

Brain affected by atherosclerosis may be more vulnerable to an ischaemic insult, and this may lead to an adverse outcome. We therefore set out to investigate the association between CCA-IMT, as a marker cerebral atherosclerosis, and functional outcome after an acute ischaemic stroke.

\section{METHODS}

\section{Cases}

During an 18 month period, 325 consecutive patients with their first ever ischaemic stroke were recruited and followed up at regular intervals up to one year post-stroke. All patients were indigenous Greeks and were admitted through the accident and emergency department into the neurology and internal medicine wards of the University Hospital of Patras (Greece). The diagnosis of stroke was made according to the WHO criteria. ${ }^{4}$ Brain imaging (by computed tomography or magnetic resonance imaging on at least one occasion within three days of admission to hospital), electrocardiography, echocardiography, and routine blood haematology and biochemistry were carried out. Patients with intracerebral or subarachnoid haemorrhage were excluded.

Data collection, risk factors, and outcome definition Demographic data and vascular risk factors were recorded on admission. The risk factors of stroke (past medical history of arterial hypertension, atrial fibrillation, ischaemic heart disease, diabetes mellitus, hypercholesterolaemia) were considered present according to standard clinical criteria. Smoking history was coded as never, previous (at least six months after smoking cessation), and current (any amount of tobacco smoked per day). Pre-stroke disability was assessed using the modified Oxford handicap scale (Rankin). ${ }^{5}$ Neurological assessments were carried out by an experienced neurologist using the Scandinavian stroke scale $(\text { SSS })^{6}$ within 24 hours of admission. Classification of the characteristics of the ischaemic lesion-that is, size, location, and side-was based on the clinical features of the presenting stroke and the brain imaging findings. Co-medication was not evaluated. During the acute stages all patients received antiplatelet treatment and none was treated with thrombolytic agents or heparins. Functional outcome was assessed at seven days from stroke onset, at discharge from hospital, and one year post-stroke using the modified Barthel activities of daily living index (BI). ${ }^{7}$ Bad outcome at seven days and discharge was defined as death or $\mathrm{BI} \leqslant 15$, and at one year post-stroke as death or BI $<18$. Outcome assessments were carried out by an investigator blind to the results of the carotid ultrasonography.

\section{Carotid ultrasonography measurements}

Of the 325 patients enrolled in the study, 284 (87\%) underwent carotid ultrasonography during their hospital admission. Carotid ultrasonography was not carried out in 41 patients ( 25 died in hospital during the early stages of stroke; in 16 patients it could not be done for technical reasons). A single experienced operator (TG) carried out all ultrasonographic investigations using an ATL HDI 3500 with a 12-5 linear transducer. The carotid arteries were first examined in the transverse plane and then longitudinally. Measurement of intima-media thickness was made on the far wall of the common carotid artery, $1.5 \mathrm{~cm}$ proximal to the bifurcation, at a point free of plaques, using a technique described elsewhere. ${ }^{8}$ The degree of carotid stenosis was categorised in three groups $(<40 \%, 40-70 \%, \geqslant 70 \%)$, according to the peak systolic velocity of the internal carotid/common carotid

Abbreviations: BI, Barthel activities of daily living index; CCA-IMT, common carotid artery intima-media thickness; NASCET, North American symptomatic carotid endarterectomy trial; SSS, Scandinavian stroke scale 
artery, following the criteria advocated in the North American symptomatic carotid endarterectomy trial (NASCET). ${ }^{9}$

\section{Statistical analysis}

The Mann-Whitney U test for two samples was used in nonparametric comparisons, and $\chi^{2}$ testing with Yates corrected probability $(p)$ values in the comparison of proportions. The odds ratios (OR) with 95\% confidence intervals (CI) were computed for an increase of 1 SD in CCA-IMT. Adjustments for confounding factors were made using multivariate logistic regression models. The study was approved by the local ethics committee.

\section{RESULTS}

Overall, 284 patients (mean (SD) age, 68.7 (12.7) years, 126 $(44 \%)$ female, median in-hospital stay nine days (range 2 to 43)) were included in the study and followed up to one year post-stroke. The general characteristics of the patients are shown in table 1.

CCA-IMT was strongly correlated with age (Pearson $r$ correlation coefficient $=0.313, \mathrm{p}<0.001$ ). CCA-IMT, after accounting for age, was correlated with a history of diabetes mellitus, ischaemic heart disease, and current smoking (Spearman's $\rho$ correlation coefficients $0.154 \quad(p=0.010)$, $0.162(p=0.006)$, and $0.186(p=0.002)$, respectively $)$. The CCA-IMT was also associated with the number of concurrent risk factors (Mann-Whitney $U$ test, $\mathrm{p}=0.036$ ), as shown in fig 1 . However, after stratifying by age group this association was significant only in the younger patients $(<65$ years, Mann-Whitney U test, $\mathrm{p}=0.045$ ).

CCA-IMT was not associated with adverse functional outcome either at discharge from hospital or at one year post-stroke in univariate analysis (OR (95\% CI): 1.24 (0.95 to 1.61 ) and 1.26 ( 1.00 to 1.58 ), respectively). After adjusting in the multivariate logistic regression analysis sequentially for age, sex, stroke severity, and pre-stroke disability; arterial hypertension, atrial fibrillation, ischaemic heart disease, diabetes mellitus, and hypercholesterolaemia in addition to the previous factors; and carotid stenosis, side and site/ extend of the lesion in addition to all previous factors, the

\begin{tabular}{lc} 
Table 1 The general characteristics of the 284 patients \\
included in the study \\
\hline Variable \\
\hline Age (mean (SD)) \\
Sex (female) \\
Initial stroke severity* (mean (SD)) \\
Pre-stroke disability (Rankin) \\
None (scores 0-1) & $68.7(126(44 \%)$ \\
Moderate (scores 2-3) & $31.4(15.7)$ \\
Severe (scores 4-5) & $235(83 \%)$ \\
History of arterial hypertension & $41(14 \%)$ \\
History of diabetes mellitus & $8(3 \%)$ \\
History of atrial fibrillation & $206(73 \%)$ \\
History of IHD & $95(33 \%)$ \\
History of hypercholesterolaemia & $72(25 \%) \dagger$ \\
Current smoking & $66(23 \%)$ \\
Side of hemispheric lesion (right) & $134(48 \%) \neq$ \\
Site/extend of lesion (large cortical/subcortical) & $140(49 \%)$ \\
Carotid stenosis & $91(32 \%)$ \\
$<40 \%$ & $217(76 \%)$ \\
$40-70 \%$ & $46(16 \%)$ \\
$\geqslant 70 \%$ & $21(7 \%)$ \\
CCA-IMT (mean (SD)) & $0.794(0.155)$ \\
\hline
\end{tabular}

Values are $n(\%)$ unless specified.

*Scandinavian stroke scale score on admission (range 0 to 58). †Information was missing in one case.

\#Information was missing in three cases.

CCA-IMT, common carotid artery intima-media thickness; IHD, ischaemic heart disease.

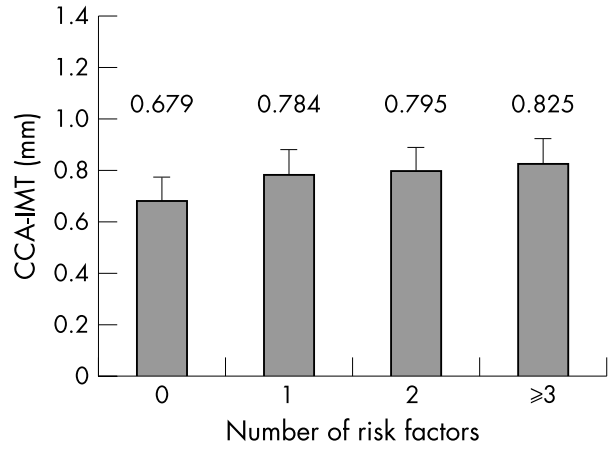

Figure 1 Common carotid artery intima-media thickness (CCA-IMT) in relation to the number of vascular risk factors.

association was still not significant (ORs 1.03 (0.73 to 1.46), 0.97 (0.68 to 1.38$)$, and 0.90 (0.61 to 1.32$)$, respectively, for outcome at discharge, and 1.17 (0.85 to 1.62$), 1.40$ (0.95 to 2.13 ), and 1.23 (0.86 to 1.75 ) for outcome at one year poststroke).

Only age and initial stroke severity were significantly associated with adverse short and long term functional outcome after correcting for the confounding factors in the multivariate logistic regression analysis (table 2). Similar results, with no significant association between CCA-IMT and bad outcome, were found when CCA-IMT was related with functional outcome at day 7 (results not shown in table 2). After categorisation of CCA-IMT into quartiles, the risk of a bad outcome for the upper three quartiles compared with the lowest quartile at discharge and at one year poststroke did not change significantly with increasing CCA-IMT.

\section{DISCUSSION}

Our hypothesis that brain affected by atherosclerosis-as indicated by increased CCA-IMT-may be more vulnerable to the ischaemic insult, and that this may lead to an adverse outcome, does not appear to be supported by the findings of this study. CCA-IMT was not associated with either short or long term functional outcome after accounting for age, sex, initial stroke severity, and pre-stroke disability. This lack of association was maintained after adjustment for other conventional stroke risk factors and lesion characteristics. Age and initial stroke severity were strong predictors of adverse functional outcome. None of the other variables investigated was associated with a bad outcome. The inclusion of the initial stroke severity in the multivariate logistic regression model may explain the lack of association between the site and extent of the lesion and functional outcome. The association of stroke risk factors with a worse functional outcome is doubtful. Only a few studies document an association with diabetes mellitus, hypercholesterolaemia, and atrial fibrillation. ${ }^{10-12}$ It is likely that the two strong outcome predictors-initial neurological deficit and age ${ }^{13}-$ obscure any possible negative effect of cerebral atherosclerosis either on the ischaemic penumbra during the early stages or on brain plasticity later on during recovery.

The carotid ultrasonography is capable of accurate measurement of intima-media thickness. ${ }^{14}$ In this study, CCAIMT measurements were strongly associated with age, history of diabetes mellitus and ischaemic heart disease, and smoking. A significant association of CCA-IMT with age and the conventional vascular risk factors has also been reported in other studies. ${ }^{15-17}$ In the present work, CCA-IMT increased with the number of concurrent risk factors, but after stratifying for age, this association was significant only for the younger group of patients. Similar results, but independent of age, have been reported by Baldassarre 
Table 2 Association of general characteristics, vascular risk factors, stroke features, and carotid ultrasonographic measurements with short and long term functional outcome in the multivariate logistic regression analysis

\begin{tabular}{lll}
\hline Variable & Outcome at discharge & $\begin{array}{l}\text { Outcome at one year post- } \\
\text { stroke }\end{array}$ \\
\hline Age $^{*}$ & $1.44(1.17$ to 1.76$)$ & $1.66(1.33$ to 2.08$)$ \\
Sex (female) $_{\text {Initial stroke severity* }}$ & $2.40(0.98$ to 5.89$)$ & $2.27(0.99$ to 5.17$)$ \\
Pre-stroke disability (Rankin) & $0.53(0.44$ to 0.63$)$ & $0.59(0.51$ to 0.68$)$ \\
History of arterial hypertension & $1.80(0.99$ to 3.24$)$ & $1.69(1.08$ to 2.65$)$ \\
History of diabetes mellitus & $2.48(0.99$ to 6.15$)$ & $0.89(0.36$ to 2.16$)$ \\
History of atrial fibrillation & $1.23(0.52$ to 2.91$)$ & $0.57(0.26$ to 1.23$)$ \\
History of IHD & $1.81(0.59$ to 5.56$)$ & $0.97(0.40$ to 2.34$)$ \\
History of hypercholesterolaemia & $0.46(0.17$ to 1.29$)$ & $0.52(0.22$ to 1.24$)$ \\
Current smoking & $0.64(0.28$ to 1.47$)$ & $0.97(0.47$ to 2.00$)$ \\
Side of hemispheric lesion (right) & $1.60(0.63$ to 4.07$)$ & $1.45(0.59$ to 3.56$)$ \\
Site/extend of lesion (large cortical/ & $0.73(0.52$ to 1.06$)$ & $0.83(0.57$ to 1.19$)$ \\
subcortical) & $1.02(0.75$ to 1.37$)$ & $0.97(0.73$ to 1.29$)$ \\
Carotid stenosis & $1.55(0.96$ to 2.51$)$ & $1.07(0.74$ to 1.55$)$ \\
CCA-IMT & $0.90(0.61$ to 1.32$)$ & $1.23(0.86$ to 1.75$)$ \\
\hline
\end{tabular}

Values are odds ratios with $95 \%$ confidence intervals.

*Age and initial stroke severity according to the Scandinavian stroke scale were computed per increase of five years and 5 points, respectively.

CCA-IMT, common carotid artery intima-media thickness, IHD, ischaemic heart disease.

et $a{ }^{18}$ a further indication that intima-media thickness is a comprehensive marker of carotid and even more widespread atherosclerosis.

There are certain aspects to be taken into account in this study. Intima-media thickness measured at the far wall of the common carotid artery, although more accurate, may be of less value than measurements at multiple carotid sites as an indicator of atherosclerosis. ${ }^{19}$ Survival bias cannot be excluded. However, patients who died before undergoing ultrasonography had suffered more severe strokes, so presumably including them in the analysis would merely increase the association of stroke severity with outcome. The duration and severity of the risk factors, as well as the use of statins, were not recorded and this may be important. Rehabilitation therapy is given to all patients while in hospital, but after discharge it is haphazard; thus it is unlikely to have biased the results. The association of CCAIMT with recurrent stroke and other vascular events has not been investigated; however, this provides scope for a study with a longer follow up period.

\section{Conclusions}

CCA-IMT was not associated with short or long term adverse functional outcomes after an ischaemic stroke. Age and initial stroke severity were the only variables that were significantly associated with a bad outcome. Adding CCAIMT in a prediction model for stroke outcome would probably not improve the power of the model.

\section{Authors' affiliations}

J Ellul, P Talelli, G Gioldasis, T Papapetropoulos, Department of

Neurology, University of Patras, Patras, Greece

G Terzis, A Chrysanthopoulou, Department of Radiology, University of Patras

Competing interests: none declared

Correspondence to: Dr J Ellul, Department of Neurology, University Hospital of Patras, Rio, Patras 26500, Greece; ellul@otenet.gr

Received 25 April 2003

Revised 11 November 2003

Accepted 11 November 2003

\section{REFERENCES}

1 Burke GL, Evans GW, Riley WA, et al. Arterial wall thickness is associated with prevalent cardiovascular disease in middle-aged adults. The Atherosclerosis Risk in Communities (ARIC) Study. Stroke 1995;26:386-91.

2 Bots ML, Hoes AW, Koudstaal PJ, et al. Common carotid intima-media thickness and risk of stroke and myocardial infarction: the Rotterdam Study. Circulation 1997;96:1432-7

3 O'Leary DH, Polak JF, Kronmal RA, et al. Carotid-artery intima and media thickness as a risk factor for myocardial infarction and stroke in older adults. Cardiovascular Health Study Collaborative Research Group. N Engl J Med 1999;340:14-22.

4 Hatano $S$, ed. Experience from a multicentre stroke register: a preliminary report. WHO Bull 1976;54:541-52.

5 Bamford JM, Sandercock PA, Warlow CP, et al. Interobserver agreement for the assessment of handicap in stroke patients. Stroke 1989;20:828.

6 Scandinavian Stroke Study Group. Multicenter trial of hemodilution in acute ischemic stroke. Results of subgroup analyses. Stroke 1988;19:464-71.

7 Collin C, Wade DT, Davies S, et al. The Barthel ADL index: a reliability study. Int Disabil Stud 1988;10:61-3.

8 Ebrahim S, Papacosta O, Whincup P, et al. Carotid plaque, intima media thickness, cardiovascular risk factors, and prevalent cardiovascular disease in men and women: the British Regional Heart Study. Stroke 1999;30:841-50.

9 Eliasziw M, Rankin RN, Fox AJ, et al. Accuracy and prognostic consequences of ultrasonography in identifying severe carotid artery stenosis. Stroke 1995;26:1747-52.

10 Megherbi SE, Milan C, Minier D, et al. Association between diabetes and stroke subtype on survival and functional outcome 3 months after stroke: data from the European BIOMED Stroke Project. Stroke 2003;34:688-94.

11 Dyker AG, Weir CJ, Lees KR. Influence of cholesterol on survival after stroke: retrospective study. BMJ 1997;314:1584-8.

12 Sandercock $\mathbf{P}$, Bamford J, Dennis $M$, et al. Atrial fibrillation and stroke: prevalence in different types of stroke and influence on early and long term prognosis (Oxfordshire community stroke project). BMJ 1992;305:1460-5.

13 Appelros P, Nydevik I, Viitanen M. Poor outcome after first-ever stroke: predictors for death, dependency, and recurrent stroke within the first year. Stroke 2003;34:122-6.

14 Wong M, Edelstein J, Wollman J, et al. Ultrasonic-pathological comparison of the human arterial wall. Verification of intima-media thickness. Arterioscler Thromb 1993;13:482-6.

15 Allan PL, Mowbray PI, Lee AJ, et al. Relationship between carotid intimamedia thickness and symptomatic and asymptomatic peripheral arterial disease. The Edinburgh Artery Study. Stroke 1997;28:348-53.

16 Sun P, Dwyer KM, Merz CN, et al. Blood pressure, LDL cholesterol, and intima-media thickness: a test of the "response to injury" hypothesis of atherosclerosis. Arterioscler Thromb Vasc Biol 2000;20:2005-10.

17 Sun Y, Lin CH, Lu CJ, et al. Carotid atherosclerosis, intima media thickness and risk factors - an analysis of 1781 asymptomatic subjects in Taiwan. Atherosclerosis 2002;164:89-94.

18 Baldassarre D, Amato M, Bondioli A, et al. Carotid artery intima-media thickness measured by ultrasonography in normal clinical practice correlates well with atherosclerosis risk factors. Stroke 2000;31:2426-30.

19 Simon A, Gariepy J, Chironi G, et al. Intima-media thickness: a new tool for diagnosis and treatment of cardiovascular risk. J Hypertens 2002;20:159-69. 